\title{
VARIACION ESTACIONAL DE LA FLORA Y VEGETACION EN LA PRECORDILLERAANDINA DE LA COMUNA DE PUTRE (I REGION DE TARAPACA, CHILE) DURANTE EL PERIODO 2002-2003
}

\author{
SEASONAL FLORISTIC AND VEGETATIONAL CHANGE IN THE \\ PRE-ANDEAN MOUNTAIN IN PUTRE COUNTY (I REGION OF \\ TARAPACA, CHILE), DURING 2002 AND 2003
}

\author{
Alejandra E. Muñoz ${ }^{1} \&$ Cristián Bonacic ${ }^{1}$ \\ ${ }^{1}$ Fauna Australis, Departamento de Ciencias Animales, Facultad de Agronomía e Ingeniería Forestal, Pontificia \\ Universidad Católica de Chile, Casilla 306, Correo 22, Santiago,Chile. aemunoz@puc.cl
}

\begin{abstract}
RESUMEN
La precordillera de la I Región de Chile $\left(18^{\circ} 15^{\prime} \mathrm{S}-69^{\circ} 33^{\prime} \mathrm{W}\right)$ es una franja que recibe efectos tanto del adyacente desierto ubicado hacia el poniente a menor altitud, como del Altiplano, el piso superior con el cual limita hacia el oriente. La vegetación en el área presenta, en consecuencia, una variación altitudinal, donde la precordillera se ubica en el segundo de cuatro pisos denominado comúnmente tolar o estepa arbustiva pre-altiplánica (sensu Gajardo). En esta zona 10 parcelas fueron muestreadas mediante el uso de transectos, en cuatro trabajos en terreno durante el año 2002 y 2003 . En ellas se investigó acerca de las formas de vida, cobertura vegetal absoluta y relativa, diversidad y riqueza de especies vegetales vasculares en un período anterior y posterior a la época de lluvias estivales (estación seca y estación húmeda). La precipitación estival del año 2002 fue 203 mm, un monto normal para la región, mientras que el año 2003 la precipitación estival fue menor e igual a $84,7 \mathrm{~mm}$ (inferior al $50 \%$ del promedio de la última década a esa fecha). La amplia mayoría de especies vegetales vasculares encontradas $(92,9 \%)$ es de origen nativo ( $\mathrm{n}=98$ especies) y sus principales formas de vida son caméfitas, nanofanerófitas y hemicriptófitas. Los resultados de los transectos presentaron patrones de estacionalidad; arrojaron mayor cobertura, riqueza de especies y mayores índices de biodiversidad durante la estación húmeda respecto a la seca. En la estación seca nanofanerófitas y caméfitas, principalmente tolas (de la voz aymara t'ola que significa arbusto), son las formas de vida más abundantes, mientras que en la estación húmeda se suman las terófitas y hemicriptófitas herbáceas. El cambio proporcional de cobertura vegetal entre estaciones húmeda y seca, así como la participación relativa de terófitas se vieron disminuidos durante el año 2003, precedido por menor precipitación estival que el año 2002.
\end{abstract}

PALABRAS ClAVEs: Altiplano, vegetación, formas de vida, lluvias estivales.

\section{ABSTRACT}

The pre-andean mountains of the northern Chile $\left(18^{\circ} 15^{\prime} \mathrm{S}-69^{\circ} 33^{\prime} \mathrm{W}\right)$ corresponds to a narrow strip which is affected by the desert at lower altitud to the west, and the Altiplano, a higher plateau at the east. The vegetation in the area also presents an altitudinal variation, and the pre-andean mountains constitutes the second of four altitudinal floors, commonly called tolar or pre-altiplano shrubland (sensu Gajardo). Ten sampling sites were studied for life forms of plants, absolute and relative vegetal cover, plant diversity and species richness of vascular plants before and after summer rainfall (dry and wet season) in four expeditions during 2002 and 2003 by using transects. The summer rainfall in 2002 was $203 \mathrm{~mm}$, considered a normal precipitation for the region, whereas the following year presented a lower amount of rain of $84.7 \mathrm{~mm}$ (less than $50 \%$ of normal summer rain for the area). The majority of the species of plants $(92,9 \%)$ were native (total $n=98$ species) and the most abundant life forms were chamaephytes, nanophanerophytes and hemicryptophytes. The results of the transects showed patterns of seasonality; they presented higher vegetal cover, species richness and biodiversity in wet season. In the dry season nanophanerophytes and chamaephytes, mainly tolas (shrubs in Aymara language), are the most abundant life forms. Whereas in the wet season therophytes and hemicryptophytes herbs also are present. The proportional change of vegetal cover between wet and dry season and the presence of terophytes community were lower in the wet season during 2003, which was dryer than 2002.

KEYwORDS: Altiplano, vegetation, life forms, summer rainfalls. 
Gayana Bot. 63(1), 2006

\section{INTRODUCCION}

Los ecosistemas del norte de Chile corresponden a ecosistemas desérticos y forman parte de una unidad geomorfológica árida mayor, comprendida entre Perú y el norte de Chile, cuya existencia es el resultado de su posición latitudinal, la corriente marina fría de Humboldt y el efecto de sombra de lluvias que ejerce la Cordillera de los Andes (Arroyo et al. 1988, Marquet et al. 1998). En el extremo norte de Chile, llámese I y II regiones, la Cordillera de los Andes pertenece a una gran unidad orográfica del macizo andino conocida como el Altiplano. Este es un sector de tierras altas de América del Sur localizado en los Andes centrales, entre $15^{\circ}$ y $27^{\circ} \mathrm{S}$ aproximadamente, y es compartido por Argentina, Bolivia, Chile y Perú (Charrier 1997). Su elevación sobre el nivel del mar, cercana a $4.000 \mathrm{~m}$, condiciona la existencia de características climáticas únicas en el continente (Aceituno 1997), las cuales afectan a las especies de fauna y flora presentes.

De ese modo, la flora y vegetación de la I Región presentan una marcada segregación según altitud y en consecuencia clima, lo cual se traduce en la existencia de pisos o cinturones vegetacionales desarrollados en un sentido longitudinal (CONAF 1981), al igual como ocurre en otros hábitat de montaña en el mundo (Cavieres et al. 2000).

La precordillera corresponde al segundo de cuatro pisos o cinturones vegetacionales ordenados de menor a mayor altitud en los Andes de la I Región, denominado comúnmente como puna (Villagrán et al. 1981, Gutiérrez et al. 1988, Arroyo et al. 1997, Negrete 1997), tolar o matorral bajo (CORFO 1982, Villagrán et al. 1982) o estepa arbustiva prealtiplánica (Gajardo 1994, Gajardo 1997, Rundel 2003). Ante la variedad de denominaciones, y concordando de este modo con Rundel (2003), se escogen en este trabajo la de tolar o estepa arbustiva prealtiplánica excluyendo el término puna debido a que ha sido considerado como más amplio para referirse a todas las comunidades vegetales que viven en el Altiplano. El tolar, ubicado entre 3.100 y $3.800 \mathrm{~m}$ de altitud, corresponde al piso más rico en especies y de mayor cobertura vegetal. Su fisionomía está dominada por formaciones arbustivas o tolares (de la voz aymara $t^{\prime}$ ola) de hojas resinosas y persistentes.

Las condiciones abióticas en las cuales se desarrollan las comunidades vegetales son rigurosas; existe una baja concentración de oxígeno y dióxido de carbono en el aire, la humedad relativa es baja y existe alta radiación solar. Además, los suelos se encuentran pobremente desarrollados y presentan una escasa disponibilidad de nutrientes (Marquet et al. 1998). La geomorfología del lugar está caracterizada por la dominancia de laderas rocosas de alta pendiente. Existen muy pocos terrenos planos.

Este ecosistema, además, constituye el hábitat de diversas especies nativas de fauna tales como guanaco (Lama guanicoe Müller), taruca o ciervo andino (Hippocamelus antisensis D'Orbigny), quirquincho de la puna (Chaetophractus nationi Thomas), la perdiz de la puna (Tinamotis pentlandii Vigors) y el picaflor del norte (Rhodopis vesper Lesson); entre ellos destaca la taruca o ciervo andino (Hippocamelus antisensis), pues presenta problemas en su estado de conservación (Glade 1993) y su presencia en Chile está ligada exclusivamente a este ecosistema (Sielfeld et al. 1988, 1999).

Los estudios realizados en la vegetación de la I Región en su mayoría realizan comparaciones a través de un gradiente altitudinal pero carecen de información acerca de lo que ocurre en un mismo piso a través de distintas estaciones de crecimiento o entre años consecutivos. Este estudio tuvo por primer objetivo distinguir la estructura y fisonomía de las distintas comunidades vegetales presentes en la zona precordillerana de la localidad de Putre y definir como varían entre estaciones de crecimiento determinadas, principalmente, por la disponibilidad hídrica. Además se observó cómo se comportaron las variaciones estacionales de cobertura vegetal entre años consecutivos.

Área DE ESTUdio

La zona de estudio se ubica en la precordillera del extremo norte de Chile en la Región de Tarapacá (I Región). Específicamente, el área de estudio se desarrolla de norte a sur en un eje que va desde el poblado de Putre $\left(18^{\circ} 11^{\prime} 52^{\prime \prime} \mathrm{S}-69^{\circ} 33^{\prime} 31^{\prime \prime} \mathrm{W}\right)$ hasta el poblado de Belén (18 $28^{\prime} 3^{\prime \prime} \mathrm{S}-6^{\circ} 30^{\prime} 54^{\prime \prime} \mathrm{W}$ ) alrededor de los 3.200 a $4.000 \mathrm{~m}$ de altitud (Fig. 1).

Según Di Castri y Hajek (1976) el área de estudio se ubica en una zona de desierto marginal de altura con una tendencia tropical, pues las lluvias accidentales tienden a ocurrir en verano. De acuerdo a la clasificación agroclimática realizada por Novoa 
y Villaseca (1989), el área de estudio se desarrolla bajo un clima desierto de altura, específicamente en los agroclimas Putre y Potrerillos. En ella el promedio menor del mes más frío oscila alrededor de $0^{\circ} \mathrm{C}$ y la máxima media del mes más cálido varía entre $10 \mathrm{a} 18^{\circ}$ C. La precipitación anual se ubica entre 50 y $200 \mathrm{~mm}$ y está concentrada principalmente en enero y febrero, debido a la influencia del ciclón continental sudamericano de características tropicales; respecto a esto último, según los datos de pluviometría entregados por las estaciones Putre y Central Chapiquiña de la Dirección General de Aguas (DGA) del Ministerio de Obras Públicas (MOP), la precipitación estival (en este estudio considerada como aquella ocurrida entre diciembre y marzo del año siguiente) concentra más del $90 \%$ de la precipitación anual. Debido a esto se puede distinguir dos estaciones de crecimiento de plantas de acuerdo a la disponibilidad hídrica del suelo: una estación seca previo a la caída de lluvias estivales y una estación húmeda de mayor cobertura y riqueza vegetal, posterior a la misma.

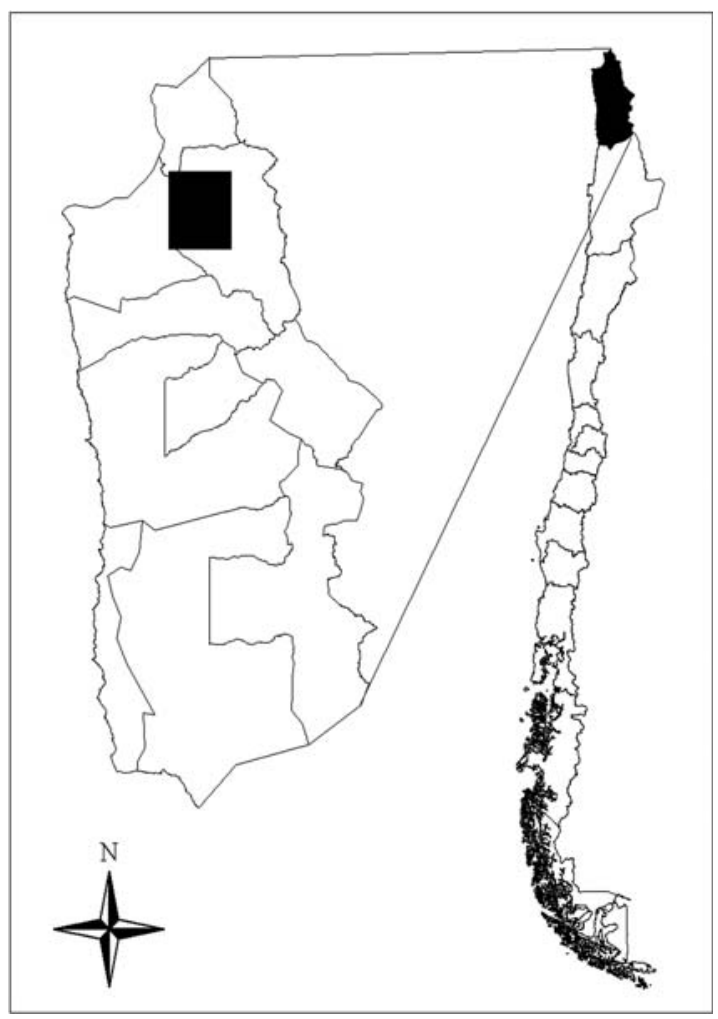

FIGURA 1: Ubicación del área de estudio.

FIGURE 1: Location of the study area.
La precordillera en esta área, conocida como la Cordillera Central de Chapiquiña, es una cadena montañosa que corresponde al borde occidental del Altiplano. Respecto a sus características edáficas, la ausencia de condiciones climáticas que posibiliten una actividad orgánica adecuada resulta en suelos de escasa evolución y delgados. Los suelos se desarrollan en condiciones de fuertes pendientes y, en forma general, son en parte litosólicos y regolíticos, de colores pardo a pardo grisáceos. Poseen texturas medias y gravosas en superficie, con cierto contenido arcilloso en profundidad. Es común la presencia de afloramientos rocosos. En esta unidad también se incluyen terrazas artificiales constituidas por suelos de quebradas y coluvios, utilizadas con fines agrícolas (CONAF 1981, 1986).

\section{MATERIALESYMETODOS}

Se realizaron cuatro expediciones al terreno, dos de ellas en el año 2002 y las dos restantes en el 2003. Durante éstas, un total de 10 parcelas de una hectárea de superficie fueron establecidas como sitios de muestreo a lo largo del área de estudio (Fig. 2). Durante el año 2002 se tomaron los datos de las parcelas uno, dos y tres en Abril (estación húmeda) y diciembre (estación seca). En el año 2003, por su parte, se tomaron los datos de las parcelas restantes (cuatro a 10) en enero (estación seca) y abril-mayo (estación húmeda). Las mediciones hechas en diciembre de 2002 y enero 2003, meses incluidos dentro del período estival, fueron consideradas como representativas de la estación seca, pues a la fecha no había caído agua, por lo cual la vegetación no había cambiado.

Los sitios escogidos eran homogéneos en cuanto no presentaban indicios de intervención antrópica ni se encontraban contiguos a caminos; estos sitios correspondían a terrenos ondulados en laderas de cerros con un rango de 18 a $53 \%$ de pendiente (Tabla I).

Las precipitaciones ocurridas en los años 2002 y 2003 presentaron notables diferencias: la precipitación estival 2001-2002 alcanzó 203 mm, mientras que la precipitación estival 2002-2003 fue de 84,7 mm. Los datos de pluviometría fueron obtenidos de la estación Putre $\left(18^{\circ} 12^{\prime} 9,7^{\prime \prime} \mathrm{S}-69^{\circ} 33^{\prime} 30,1^{\prime \prime} \mathrm{W}\right)$ para las parcelas medidas más al norte (uno, dos y tres) y de la estación Central Chapiquiña (18 $22^{\prime} 35,5^{\prime \prime} \mathrm{S}-69^{\circ} 32^{\prime} 47,8^{\prime \prime} \mathrm{W}$ ) para las parcelas restantes, del Centro de Información de Recursos Hídricos (DGA-MOP). 
Gayana Bot. 63(1), 2006

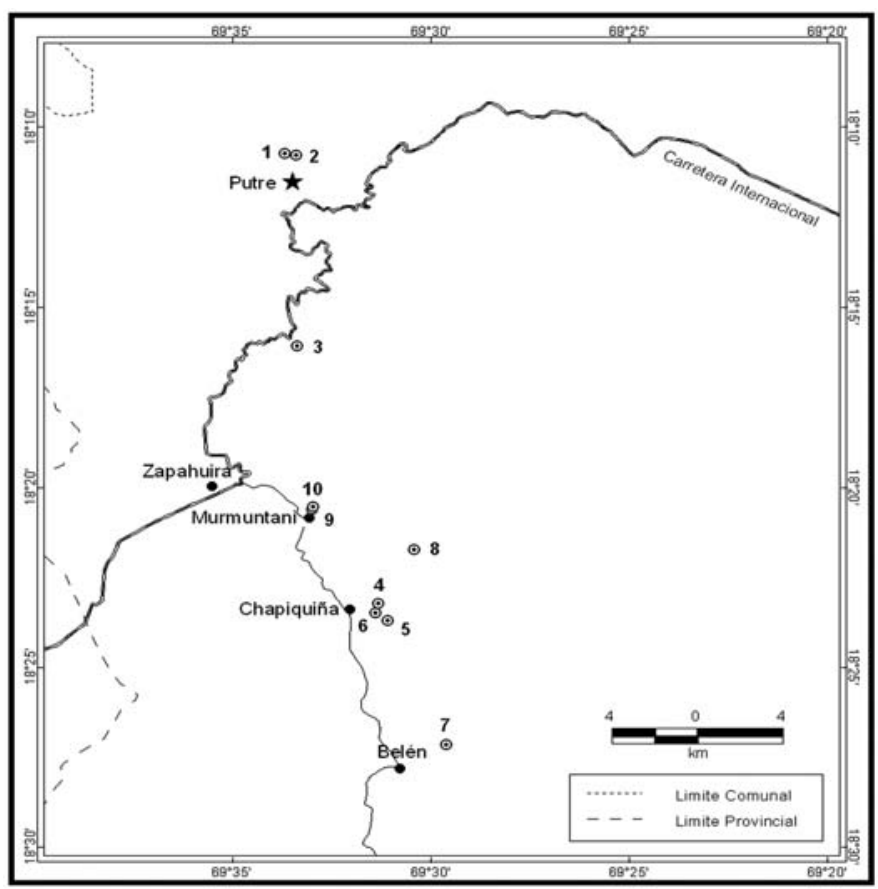

Figura 2: Ubicación de parcelas de muestreo.

FIGURE 2: Location of the sampling sites.

TABLA I. Ubicación y descripción de las parcelas de muestreo en el área de estudio.

TABLE I. Location and description of the sampling sites in the study area.

\begin{tabular}{|c|c|c|c|c|c|}
\hline $\mathrm{N}^{\mathrm{o}}$ & sector & $\begin{array}{l}\text { ubicacion } \\
\text { geográfica }\end{array}$ & $\begin{array}{c}\text { pendiente } \\
(\%)\end{array}$ & $\begin{array}{c}\text { altitud } \\
\text { (ms.n.m.) }\end{array}$ & exposición \\
\hline 1 & Putre & $\begin{array}{l}18^{\circ} 10^{\prime} 43,8^{\prime \prime} \mathrm{S} \\
69^{\circ} 33^{\prime} 38,9^{\prime \prime} \mathrm{W}\end{array}$ & 31 & 3790 & NE \\
\hline 2 & Putre & $\begin{array}{l}18^{\circ} 10^{\prime} 47,4^{\prime \prime} \mathrm{S} \\
69^{\circ} 33^{\prime} 22,5^{\prime \prime} \mathrm{W}\end{array}$ & 24 & 3861 & NW \\
\hline 3 & Zapahuira & $\begin{array}{l}18^{\circ} 16^{\prime} 5,6^{\prime \prime} \mathrm{S} \\
69^{\circ} 33^{\prime} 21,7,7^{\prime \prime} \mathrm{W}\end{array}$ & 32 & 3615 & NW \\
\hline 4 & Chapiquiña & $\begin{array}{l}18^{\circ} 23,14,2 " \mathrm{~S} \\
69^{\circ} 31^{\prime} 18,3, \mathrm{~W}\end{array}$ & 18 & 3599 & W \\
\hline 5 & Chapiquiña & $\begin{array}{l}18^{\circ} 23^{\prime} 42,2, ' \mathrm{~S} \\
69^{\circ} 31^{\prime} 3,5, \mathrm{~W}\end{array}$ & 18 & 3625 & SW \\
\hline 6 & Chapiquiña & $\begin{array}{l}18^{\circ} 21^{\prime} 43,9 " \mathrm{~S} \\
69^{\circ} 31^{\prime} 22,1^{\prime \prime} \mathrm{W}\end{array}$ & 53 & 3547 & SW \\
\hline 7 & Belén & $\begin{array}{c}18^{\circ} 27^{\prime} 9,5 \text { ' } \mathrm{S} \\
69^{\circ} 29^{\prime} 35,4 \text { " } \mathrm{W}\end{array}$ & 19 & 3604 & NW \\
\hline 8 & Belén & $\begin{array}{l}18^{\circ} 21^{\prime} 44^{\prime \prime} \mathrm{S} \\
69^{\circ} 30^{\prime} 23,7^{\prime \prime} \mathrm{W}\end{array}$ & 30 & 3500 & W \\
\hline 9 & Murmuntani & $\begin{array}{l}18^{\circ} 20^{\prime} 37,2^{\prime \prime} \mathrm{S} \\
69^{\circ} 32^{\prime} 57,6^{\prime \prime} \mathrm{W}\end{array}$ & 24 & 3638 & NW \\
\hline 10 & Murmuntani & $\begin{array}{l}18^{\circ} 20^{\prime} 32,99^{\prime \prime} \mathrm{S} \\
69^{\circ} 32^{\prime} 56,2^{\prime \prime} \mathrm{W}\end{array}$ & 40 & 3644 & SW \\
\hline
\end{tabular}


Variación estacional en vegetación andina. MuÑOz, A. \& C. BonACic.

Con el fin de obtener un listado florístico del área de estudio, se recolectó una muestra de cada especie de planta vascular distinguida, tanto en las parcelas como en los recorridos por el terreno. Estos ejemplares fueron identificados y depositados en un herbario en dependencias de la Facultad de Agronomía e Ingeniería Forestal, Pontificia Universidad Católica de Chile.

Para cada especie se asignaron nombre científico, familia, forma de vida, condición de especie autóctona o alóctona de Chile y tipo biológico (Tabla II); respecto a este último, Godron et al. (1968) clasifican la vegetación en cuatro tipos biológicos fundamentales, los cuales incluyen a un grupo de especies con características convergentes en cuanto a su forma y comportamiento (en Ettiene y Prado 1982). Estos tipos son: herbáceo, leñoso bajo (de altura inferior a $2 \mathrm{~m}$ ), leñoso alto (de altura superior a $2 \mathrm{~m}$ ) y suculento. Las formas de vida de las especies se asignaron según la clasificación propuesta por el botánico C. Raunkiaer (citado por Kent \& Cooker 1992). La nomenclatura y el carácter autóctono o alóctono de Chile de las especies se basó principalmente en Marticorena (1990) y Marticorena y Quezada (1985), excepto para tres familias: Cactaceae que se basó en Hunt (1999) y Hoffmann \& Walter (2004), Poaceae donde se consultó a Soreng (2003) y Soreng \& Pennington (2003), e Iridaceae que siguió a Rodríguez \& Marticorena (2000). La categoría Poaceae fue usada para denominar especies gramíneas que no presentaban órganos reproductivos, lo cual inhabilita su identificación; por consiguiente, esta categoría podría agrupar más de una especie, lo cual lo hace un elemento no comparable; en la mayoría de los casos (sobre el $80 \%$ ) estas Poaceae correspondían a gramíneas comúnmente llamadas coirones.

Para estimar la abundancia de las especies en las comunidades vegetales se usó el método de puntos en la línea (Line Point Method), por ser considerado un método que entrega una estimación confiable de las medias poblacionales en zonas de vegetación arbustiva y es eficiente en el uso del tiempo (Heady et al. 1959). En cada parcela se realizaron tres transectos lineales de $50 \mathrm{~m}$ de largo divididos en intervalos cada $50 \mathrm{~cm}$. En cada punto se anotó la presencia (o ausencia) de una determinada especie. A partir de los datos obtenidos de los transectos se estimó: a) cobertura vegetal absoluta de cada parcela, definida como la proporción de la superficie del suelo cubierto por la proyección perpendicular de las especies vegetales; b) cobertura relativa a una especie o grupo de especies; y c) índice de biodiversidad de Shannon $(\mathrm{H})$, estimado como:

$$
h=-\sum_{i=1}^{S}(n i / n * \ln (n i / n))
$$

donde $n_{i}$ : número de individuos pertenecientes a la $i$ ésima especie, $n$ : número de individuos de la muestra y $S$ : número de especies en la muestra.

Los transectos son usados comúnmente para investigar cambios en la vegetación a través de un gradiente ambiental o a través de diferentes hábitat (Sutherland 1996); en este caso particular fueron comparados para cada parcela los resultados entre el período que antecede a las lluvias y el período posterior a las lluvias estivales. De este modo, para comparar la cobertura vegetal de las parcelas entre estaciones se utilizó la prueba no paramétrica de Wilcoxon, únicamente en las mediciones del año $2003(\mathrm{n}=7)$, pues durante el año 2002 el tamaño muestral $(n=3)$ es demasiado reducido para aplicar la prueba.

Adicionalmente, se aplicó la prueba por rangos de Spearman para investigar si el cambio en cobertura vegetal de los sitios de muestreo se relacionaba con la riqueza de especies presentes en los transectos.

Para investigar sobre el comportamiento interanual, se comparó la disminución porcentual promedio de cobertura entre el período posterior a las lluvias y el previo a la caída de las mismas, entre los años 2002 y 2003, utilizando la prueba $\mathrm{t}$.

Por último, unidades vegetacionales homogéneas fueron distinguidas en función de las características estructurales y las especies dominantes presentes en ellas de acuerdo a la metodología propuesta en la Carta de Ocupación de Tierras (Etienne \& Prado 1982); este método ha sido usado en otras investigaciones para describir la vegetación altoandina en la Provincia de Parinacota (Troncoso 1983). De acuerdo a ella, las formaciones vegetacionales se clasifican según la estratificación y cobertura de sus componentes. Para el área de estudio, como fue mencionado, se distinguieron cuatro tipos biológicos fundamentales: leñoso alto (LA), leñoso bajo (LB), herbáceo (H) y suculento (S). Así, las formaciones vegetacionales fueron denominadas de acuerdo a la dominancia de uno o más tipos biológicos categorizados según su estratificación y grado de cubrimiento del suelo, en escalas previamente definidas. Adicionalmente, en cada tipo biológico constituyente de una formación vegetacional dada, se identificaron las especies más representativas. En general, tales especies debieran alcanzar una cobertura al menos mayor o igual al $10 \%$ (Etienne \& Prado 1982). 
Gayana Bot. 63(1), 2006

TABLA II. Inventario de especies vegetales vasculares clasificadas según forma de vida, tipo biológico y origen geográfico.

TABLE II. List of vascular plant classiffied by life form, biologic type and geographic procedence.

\begin{tabular}{|c|c|c|c|c|}
\hline \multirow{2}{*}{$\frac{\text { DIVISION }}{\text { Familia }}$} & \multicolumn{4}{|l|}{ CLASE } \\
\hline & Especie & Forma de vida & Tipo biológico & $\begin{array}{l}\text { Origen } \\
\text { geográfico }\end{array}$ \\
\hline \multirow{5}{*}{$\begin{array}{l}\text { POLYPODIOPHYTA } \\
\text { Adiantaceae }\end{array}$} & \multicolumn{4}{|l|}{ POLYPODIOPSIDA } \\
\hline & \multicolumn{4}{|l|}{ Cheilanthes cf. bonariensis (Willd.) } \\
\hline & Proctor & Hemicriptófita & herbáceo & naturalizado \\
\hline & Notholaena nivea (Poir.) Desv. & Hemicriptófita & herbáceo & nativo \\
\hline & Pellaea ternifolia (Cav.) Link & Hemicriptófita & herbáceo & nativo \\
\hline PINOPHYTA & \multicolumn{4}{|l|}{ GNETOPSIDA } \\
\hline Ephedraceae & Ephedra breana Phil. & Caméfita & herbáceo & nativo \\
\hline MAGNOLIPHYTA & \multicolumn{4}{|l|}{ MAGNOLIOPSIDA } \\
\hline Apiaceae & Bowlesia tropaeolifolia Gillies et Hook. & Terófita & herbáceo & nativo \\
\hline Asclepiadaceae & Philibertia rahmeri (Phil.) Malme & Liana & herbáceo & nativo \\
\hline \multirow[t]{31}{*}{ Asteraceae } & \multicolumn{4}{|l|}{ Ambrosia artemisioides Meyen et } \\
\hline & Walp. & Caméfita & leñoso bajo & nativo \\
\hline & Baccharis boliviensis (Wedd.) Cabrera & Caméfita & leñoso bajo & nativo \\
\hline & Baccharis santelicis Phil. & Caméfita & leñoso bajo & nativo \\
\hline & Baccharis scandens (Ruiz et Pav.) Pers. & Nanofanerófita & leñoso bajo & nativo \\
\hline & Bidens triplinervia Kunth & Caméfita & herbáceo & nativo \\
\hline & Chuquiraga spinosa Less. & Nanofanerófita & leñoso bajo & nativo \\
\hline & Conyza deserticola Phil. & Hemicriptófita & herbáceo & nativo \\
\hline & Coreopsis suaveolens Sherff & Caméfita & leñoso bajo & nativo \\
\hline & Diplostephium cinereum Cuatrec. & Nanofanerófita & leñoso bajo & nativo \\
\hline & Diplostephium meyenii Wedd. & Nanofanerófita & leñoso bajo & nativo \\
\hline & Gnaphalium glandulosum (Walp.) Klatt & Caméfita & herbáceo & nativo \\
\hline & Grindelia tarapacana Phil. & Caméfita & leñoso bajo & nativo \\
\hline & \multicolumn{4}{|l|}{ Lophopappus tarapacanus (Phil.) } \\
\hline & Cabrera & Nanofanerófita & leñoso bajo & nativo \\
\hline & Mutisia acuminata Ruiz et Pav. & Nanofanerófita & leñoso bajo & nativo \\
\hline & Mutisia hamata Reiche & Liana & herbáceo & nativo \\
\hline & \multicolumn{4}{|l|}{ Ophryosporus pinifolius (Phil.) } \\
\hline & R.M.King \& H.Rob. & Nanofanerófita & leñoso bajo & nativo \\
\hline & \multicolumn{4}{|l|}{ Parastrephia lepidophylla (Wedd.) } \\
\hline & Cabrera & Nanofanerófita & leñoso bajo & nativo \\
\hline & Plazia daphnoides Wedd. & Nanofanerófita & leñoso bajo & nativo \\
\hline & Polyachyrus sphaerocephalus D.Don & Caméfita & herbáceo & nativo \\
\hline & Senecio behnii Ricardi et Martic. & Nanofanerófita & leñoso bajo & nativo \\
\hline & Senecio ctenophyllus Phil. & Nanofanerófita & herbáceo & nativo \\
\hline & \multicolumn{4}{|l|}{ Senecio olivaceobracteatus Ricardi et } \\
\hline & Martic. & Nanofanerófita & leñoso bajo & nativo \\
\hline & Senecio sp. & Nanofanerófita & leñoso bajo & nativo \\
\hline & Tagetes multiflora Kunth & Terófita & herbáceo & nativo \\
\hline & Viguiera pazensis Rusby & Caméfita & herbáceo & nativo \\
\hline & Villanova robusta Phil. & Caméfita & no determinado & nativo \\
\hline \multirow[t]{5}{*}{ Brassicaceae } & Cremolobus chilensis (Lag. ex DC.) DC. & Terófita & herbáceo & nativo \\
\hline & Descurainia stricta (Phil.) Prantl ex Reiche & Terófita & herbáceo & nativo \\
\hline & Draba gilliesi Hook. et Arn. & Caméfita & herbáceo & nativo \\
\hline & Lepidium rahmeri Phil. & Hemicriptófita & herbáceo & nativo \\
\hline & Sisymbrium sp. & Hemicriptófita & herbáceo & \\
\hline \multirow{2}{*}{ Caesalpinaceae } & \multicolumn{4}{|l|}{ Senna birostris (Dombey ex Vogel) } \\
\hline & $\begin{array}{l}\text { H.S.Irwin et Barneby var. arequipensis } \\
\text { (Meyen ex Vogel) H.S.Irwin et Barneby }\end{array}$ & Nanofanerófita & leñoso bajo & nativo \\
\hline
\end{tabular}


Variación estacional en vegetación andina. MuÑoz, A. \& C. BonACIC.

Continuación Tabla II.

\begin{tabular}{|c|c|c|c|c|}
\hline \multirow{2}{*}{$\frac{\text { DIVISION }}{\text { Familia }}$} & \multicolumn{4}{|l|}{ CLASE } \\
\hline & Especie & Forma de vida & Tipo biológico & $\begin{array}{l}\text { Origen } \\
\text { geográfico }\end{array}$ \\
\hline \multirow[t]{17}{*}{ Cactaceae } & \multicolumn{4}{|c|}{ Corryocactus brevistylus (K.Schum. ex Vaupel) } \\
\hline & Britton et Rose & Nanofanerófita & suculento & nativo \\
\hline & \multicolumn{4}{|c|}{ Cumulopuntia boliviana (Salm-Dyck) } \\
\hline & \multicolumn{4}{|c|}{ Cumulopuntia sphaerica (C.F.Först.) } \\
\hline & E.F.Anderson & Caméfita & suculento & nativo \\
\hline & Echinopsis sp. & Caméfita & suculento & nativo \\
\hline & \multicolumn{4}{|l|}{ Haageocereus fascicularis (Meyen) } \\
\hline & F.Ritter & Caméfita & suculento & endémico \\
\hline & Neowerdermannia chilensis Backeb. & Caméfita & suculento & nativo \\
\hline & Opuntia atacamensis Phil. & Caméfita & suculento & endémico \\
\hline & \multicolumn{4}{|l|}{ Oreocereus hempelianus (Gürke) } \\
\hline & D.Hunt & Caméfita & suculento & nativo \\
\hline & \multicolumn{4}{|l|}{ Oreocereus leucotrichus (Phil.) } \\
\hline & Wagenkn. & Nanofanerófita & suculento & nativo \\
\hline & Oreocereus variicolor Backeb. & Nanofanerófita & suculento & nativo \\
\hline & Tunilla soehrensii (Britton et Rose) & & & \\
\hline & D.R.Hunt et J.Iliff & Caméfita & suculento & nativo \\
\hline \multirow[t]{3}{*}{ Caryophyllaceae } & \multicolumn{4}{|l|}{ Cardionema ramosissimum (Weinm.) } \\
\hline & A.Nelson et J.F.Macbr. & Hemicriptófita & herbáceo & nativo \\
\hline & Spergularia fasciculata Phil. & Caméfita & herbáceo & nativo \\
\hline \multirow[t]{2}{*}{ Chenopodiaceae } & Chenopodium ambrosioides L. & Terófita & herbáceo & nativo \\
\hline & Chenopodium petiolare Kunth & Terófita & herbáceo & nativo \\
\hline \multirow[t]{7}{*}{ Fabaceae } & Adesmia spinosissima Meyen & Nanofanerófita & leñoso bajo & nativo \\
\hline & Adesmia verrucosa Meyen ex Vogel & Nanofanerófita & leñoso bajo & nativo \\
\hline & Astragalus sp. & $\begin{array}{l}\text { no } \\
\text { determinado }\end{array}$ & herbáceo & nativo \\
\hline & $\begin{array}{l}\text { Dalea pennellii (J.F.Macbr.) J.F.Macbr. } \\
\text { var. chilensis Barneby }\end{array}$ & $\begin{array}{l}\text { no } \\
\text { determinado }\end{array}$ & herbáceo & nativo \\
\hline & Lupinus oreophilus Phil. & Nanofanerófita & leñoso bajo & nativo \\
\hline & Medicago lupulina $\mathrm{L}$. & Terófita & herbáceo & naturalizado \\
\hline & Melilotus indicus (L.) All. & Nanofanerófita & leñoso bajo & naturalizado \\
\hline Geraniaceae & Erodium cicutarium (L.) L'Hér. ex Aiton & Terófita & herbáceo & naturalizado \\
\hline \multirow[t]{2}{*}{ Krameriaceae } & \multicolumn{4}{|l|}{ Krameria lappacea (Dombey) } \\
\hline & Burdet et B.B.Simpson & Caméfita & leñoso bajo & nativo \\
\hline Labiateae & Satureja sp. & Nanofanerófita & leñoso bajo & nativo \\
\hline Ledocarpaceae & Balbisia microphylla (Phil.) Reiche & Nanofanerófita & leñoso bajo & nativo \\
\hline Loasaceae & Nasa cf. urens (Jacq.) Weigend & Terófita & herbáceo & nativo \\
\hline \multirow[t]{3}{*}{ Malvaceae } & \multicolumn{4}{|l|}{ Fuertesimalva chilensis (A.Braun et } \\
\hline & C.D.Bouché) Fryxell & Terófita & herbáceo & nativo \\
\hline & Tarasa aff. operculata (Cav.) Krapov. & Caméfita & leñoso bajo & nativo \\
\hline Nyctaginaceae & Boerhavia diffusa $\mathrm{L}$. & Hemicriptófita & herbáceo & nativo \\
\hline \multirow[t]{3}{*}{ Plantaginaceae } & Plantago hispidula Ruiz et Pav. & Terófita & herbáceo & nativo \\
\hline & Plantago lanceolata $\mathrm{L}$. & Hemicriptófita & herbáceo & naturalizado \\
\hline & \multicolumn{4}{|l|}{$\begin{array}{l}\text { Plantago aff. sericea Ruiz et Pav. ssp. } \\
\text { sericans (Pilger) Rahn }\end{array}$} \\
\hline Polemoniaceae & Microsteris gracilis (Hook.) Greene & Terófita & herbáceo & nativo \\
\hline Polygonaceae & Muehlenbeckia hastulata (Sm.) I.M.Johnst. & Nanofanerófita & leñoso bajo & nativo \\
\hline Portulacaceae & Portulaca philippii I.M.Johnst. & Caméfita & herbáceo & nativo \\
\hline \multirow[t]{2}{*}{ Rosaceae } & \multicolumn{3}{|l|}{ Tetraglochin cristatum (Britton) } & nativo \\
\hline & Rothm. & Caméfita & leñoso bajo & nativo \\
\hline Santalaceae & Quinchamalium parviflorum Phil. & Terófita & herbáceo & nativo \\
\hline
\end{tabular}


Gayana Bot. 63(1), 2006

Continuación Tabla II.

\begin{tabular}{|c|c|c|c|c|}
\hline DIVISION & CLASE & & & \\
\hline Familia & Especie & Forma de vida & Tipo biológico & $\begin{array}{l}\text { Origen } \\
\text { geográfico }\end{array}$ \\
\hline \multirow[t]{2}{*}{ Scrophulariaceae } & Bartsia cf. peruviana Walp. & Caméfita & herbáceo & nativo \\
\hline & Calceolaria inamoena Kraenzl. & Nanofanerófita & leñoso bajo & nativo \\
\hline \multirow[t]{5}{*}{ Solanaceae } & $\begin{array}{l}\text { Dunalia spinosa (Meyen) Dammer } \\
\text { Fabiana ramulosa (Wedd) Hunz, et }\end{array}$ & Nanofanerófita & leñoso bajo & nativo \\
\hline & Barboza & Nanofanerófita & leñoso bajo & nativo \\
\hline & Lycianthes lycioides (L.) Hassl. & Nanofanerófita & leñoso bajo & nativo \\
\hline & Lycopersicum chilense Dunal & Caméfita & leñoso bajo & nativo \\
\hline & Salpichroa sp. & $\begin{array}{l}\text { no } \\
\text { determinado }\end{array}$ & leñoso bajo & nativo \\
\hline \multirow[t]{4}{*}{ Verbenaceae } & $\begin{array}{l}\text { Junellia arequipensis (Botta) Botta } \\
\text { Junellia seriphioides (Gillies et }\end{array}$ & Caméfita & leñoso bajo & nativo \\
\hline & Hook.) Moldenke & Nanofanerófita & leñoso bajo & nativo \\
\hline & Verbena gynobasis Wedd. & Caméfita & leñoso bajo & nativo \\
\hline & Verbena hispida Ruiz et Pav. & Geófita & leñoso bajo & nativo \\
\hline \multirow{3}{*}{$\begin{array}{l}\text { MAGNOLIPHYTA } \\
\text { Iridaceae }\end{array}$} & LILIOPSIDA & & & \\
\hline & Olsynium scirpoideum (Poepp.) & & & \\
\hline & Goldblatt & Geófita & herbáceo & nativo \\
\hline \multirow[t]{9}{*}{ Poaceae } & Bromus catharticus Vahl & Hemicriptófita & herbáceo & nativo \\
\hline & Cortaderia atacamensis (Phil.) Pilger & Hemicriptófita & herbáceo & nativo \\
\hline & Eragrostis weberbaueri Pilger & Hemicriptófita & herbáceo & nativo \\
\hline & Festuca orthophylla Pilger & Hemicriptófita & herbáceo & nativo \\
\hline & Jarava lepostachya (Griseb.) F.Rojas & Hemicriptófita & herbáceo & nativo \\
\hline & Muhlenbergia peruviana (P.Beauv.) Steud. & Terófita & herbáceo & nativo \\
\hline & Nassella pubiflora (Trin. et Rupr.) E.Desv. & Caméfita & herbáceo & nativo \\
\hline & Poa sp. & Hemicriptófita & herbáceo & \\
\hline & Stipa sp. & Hemicriptófita & herbáceo & $\begin{array}{l}\text { determinado } \\
\text { nativo }\end{array}$ \\
\hline
\end{tabular}

\section{RESULTADOS}

FLORA

Un total de 98 especies vegetales vasculares fueron distinguidas en el área de estudio; de ese total, 90 fueron reconocidas a nivel de especie, con cinco de ellas descritas en categorías infraespecíficas, y las ocho restantes quedaron reconocidas hasta el nivel de género. Veinte y nueve familias botánicas conforman el listado florístico (Tabla II). La división Magnoliophyta (Angiospermas) es la más abundante con 27 familias y, dentro de esta división, la clase Magnoliopsida (Dicotiledóneas) contiene 25 de ellas. Las familias con mayor número de especies son Asteraceae (26 especies), Cactaceae (11) y Poaceae (nueve).

Las tres formas de vida más frecuentes en la flora son, en orden descendente, caméfitas, nanofanerófitas y hemicriptófitas con una participación en conjunto superior al $75 \%$ (Fig. 3).

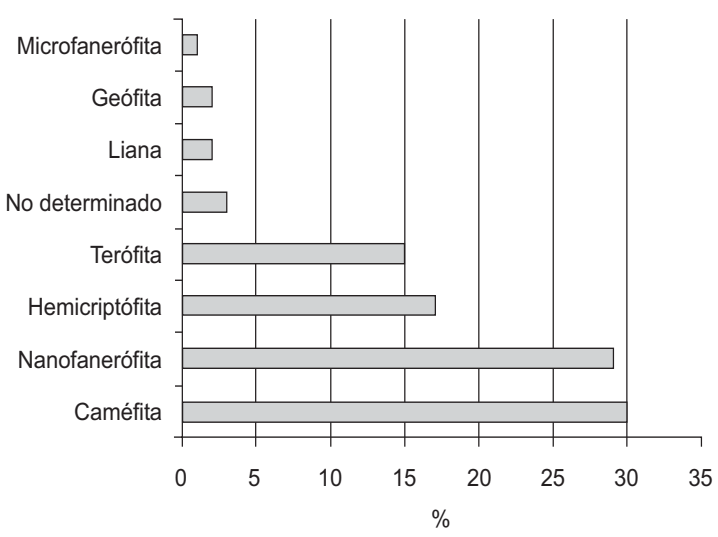

FIgURA 3: Participación de las formas de vida en la flora del área de estudio.

FIGURE 3: Relative composition of life forms in the flora of the study area. 
Del total de las especies vegetales vasculares registradas, 91 correspondieron a especies nativas $(92,9 \%)$, dos de las especies nativas correspondieron además a especies endémicas de Chile (2\%); cinco especies fueron categorizadas como especies naturalizadas $(5,1 \%)$ y dos $(2 \%)$ no fueron determinadas por estar sólo hasta la categoría de géneros cuyas especies en Chile no son sólo nativas. Las especies naturalizadas son: Cheilanthes cfr. bonariensis (Adiantaceae), Medicago lupulina y Melilotus indicus (Fabaceae), Erodium cicutarium (Geraniaceae) y Plantago lanceolata (Plantaginaceae). La primera de ellas, una Pteridofita (helecho), no está plenamente reconocida pues se recomienda confrontar con otras muestras y en el área hay otras especies del género Cheilanthes citadas (Gajardo 1997). Tanto Medicago lupulina como Melilotus indicus fueron vistas asociadas a bordes de quebradas en sustratos arenosos y húmedos. Las últimas especies, Erodium cicutarium y Plantago lanceolata son malezas de gran distribución en Chile, y en el área de estudio se vieron únicamente en lugares intervenidos por el hombre. Las especies no determinadas fueron: Sisymbrium sp. (Brassicaceae) y Poa sp. (Poaceae). Las dos especies endémicas a Chile, Haageocereus fascicularis y Opuntia atacamensis, pertenecen a la familia Cactaceae.

En conjunto, la flora del área de estudio presenta tanto géneros endémicos de los Andes de Sudamérica, tales como Polylepis, Chuquiraga, Parastrephia y Oreocereus, así como también se presentan géneros de las familias más numerosas de plantas (Asteraceae, Poaceae, Fabaceae, Brassicaceae, etc.) que están representados en forma cosmopolita (e.g. Senecio, Stipa, Lupinus y Descurainia). No existen, eso sí, géneros endémicos a Chile, pues esta región ecológica está también presente en la zona andina de las vecinas naciones de Argentina, Bolivia y Perú.

\section{CoberTuRA VEgetAL}

La cobertura vegetal absoluta mostró variaciones durante las distintas estaciones de crecimiento. Durante el año 2002, en las mediciones tomadas posterior a la caída de lluvias, las parcelas promediaron una cobertura vegetal igual a (media \pm DE) $64 \pm 6,1 \%$. Posteriormente y previo al próximo período de precipitaciones, la cobertura vegetal absoluta promedio de las parcelas disminuyó a 36,5 $\pm 3,7 \%$. Durante el 2003, por su parte, la cobertura vegetal absoluta promedió $69,7 \pm 7,9$ en la estación húmeda, mientras que en la estación seca fue inferior con un valor de 54,8 $\pm 13,5$. Para estas mediciones, realizadas durante el año 2003, la prueba de Wilcoxon indica que existe evidencia de que la cobertura vegetal varía según estación del año ( $T=1, n=7 ; P=0,025)$. En las Tablas III y IV se muestra el detalle de cobertura vegetal por especie, según estación y año de muestreo.

Según los datos de cobertura relativa de cada especie vegetal, y luego de la asignación de su forma de vida, se calculó la abundancia de las formas de vida en las mediciones realizadas durante la estación húmeda y durante la estación seca (Tablas III y IV). Al comparar la abundancia de las formas de vida durante las estaciones de crecimiento (ver Tabla V), lo primero que destaca es que las terófitas, en otras palabras, las hierbas, están ausentes en los transectos realizados durante la estación seca. En cuanto a la representatividad, las principales formas de vida presentes durante la estación seca son perennes, nanofanerófitas y caméfitas. La suma de ambas categorías en esa estación fue siempre superior al $50 \%$. Sin embargo, debe tenerse en cuenta que la participación de hemicriptófitas está subestimada en ambas estaciones, pues las especies de la categoría Poaceae no aparecen con una forma de vida asignada, pero como ya fue mencionado, la mayor parte de los casos correspondían a gramíneas comúnmente llamadas coirones. La parcela 10, en donde nanofanerófitas y caméfitas están menos representadas, corresponde a un bosquete de queñoa con arbustos al igual que el caso de la parcela 3 , de ahí que sólo ellas tengan el componente de microfanerófitas, aunque con distinta abundancia.

Respecto a la variación interanual de cobertura vegetal, la disminución porcentual de la cobertura vegetal entre la estación húmeda y previo a caída de lluvias estivales fue mayor en promedio durante el año 2002 (42,5\%) que en el 2003 (22,02\%). Eso sí, esta diferencia no fue estadísticamente significativa $(\mathrm{t}=1,06 ; \mathrm{gl}=8 ; \mathrm{P}=0,025)$. En cuanto a las formas de vida destaca el caso de las terófitas. Únicamente en las mediciones realizadas durante la estación húmeda aparece esta forma de vida. Las terófitas fueron notoriamente más abundantes en los transectos medidos durante el año $2002(42,1 \pm 17,9$ $\%$ ), respecto del año $2003(16,8 \pm 10,8 \%$ ) (ver detalle en Figuras 4 y 5). 
TABLA III. Cobertura vegetal absoluta y relativa (\%), riqueza de especies e índice de biodiversidad de Shannon en las parcelas de muestreo durante la estación húmeda.

TABLE III. Absolute and relative vegetal cover (\%), species richness and Shannon index of biodiversity in samplyng sites during wet season.

\begin{tabular}{|c|c|c|c|c|c|c|c|c|c|c|}
\hline \multirow[b]{2}{*}{ Especie / Parcela } & \multicolumn{3}{|c|}{ Año 2002} & \multirow[b]{2}{*}{4} & \multicolumn{4}{|c|}{ Año 2003} & \multirow[b]{2}{*}{9} & \multirow[b]{2}{*}{10} \\
\hline & 1 & 2 & 3 & & 5 & 6 & 7 & 8 & & \\
\hline Plantago hispidula & 24,8 & 53,6 & 6,3 & 28,1 & 1,1 & 3,1 & 16,1 & 8,1 & 18,2 & 12,3 \\
\hline Baccharis boliviensis & 14,3 & 1,3 & 8,6 & 55,1 & 8,2 & 4,6 & 10,7 & 24,9 & 7,1 & 10,5 \\
\hline Fabiana ramulosa & 6,0 & 1,8 & 20,3 & & 17,6 & 14,4 & 25,4 & 18,1 & 1,0 & 0,5 \\
\hline Diplostephium meyenii & & 16,1 & 3,1 & 2,3 & & 13,9 & 23,4 & 10,4 & 19,7 & 3,6 \\
\hline pastos & 21,1 & 3,6 & 9,4 & 3,0 & 13,2 & 9,2 & 2,4 & 10,4 & 5,6 & 11,8 \\
\hline Parastrephia lepidophylla & & & & & 40,7 & & & & & \\
\hline Lophopappus tarapacanus & & & & & & 1,0 & 1,0 & 7,2 & 18,2 & 1,5 \\
\hline Plantago sericea & & 1,8 & 9,4 & & & & 9,8 & & 1,0 & 7,3 \\
\hline Microsteris gracilis & 8,3 & & 3,1 & 1,8 & 6,0 & 1,5 & & & 5,6 & 1,4 \\
\hline Polylepis rugulosa & & 9,4 & & & & & & & & 16,8 \\
\hline Baccharis santelicis & & 0,5 & 7,0 & & 2,2 & & 0,5 & 3,6 & & 12,3 \\
\hline Verbena gynobasis & & & & & & 18,0 & & 0,5 & 1,0 & 0,5 \\
\hline Cumulopuntia sphaerica & & & 3,6 & 1,7 & 2,6 & 2,4 & 4,1 & 2,5 & 0,9 & \\
\hline Stipa sp. & 0,8 & & & 0,6 & 4,4 & 3,6 & & 1,8 & 6,1 & \\
\hline Cremolobus chilensis & & 1,8 & 14,1 & & & & & & & \\
\hline Adesmia spinosissima & 0,8 & & & & 3,3 & 0,5 & 4,9 & 1,8 & 1,0 & 2,7 \\
\hline Muhlenbergia peruviana & & 3,6 & & & & & & & 7,1 & 2,3 \\
\hline Senecio sp. & & & & 0,6 & 0,6 & 7,7 & 1,0 & & 0,5 & 0,9 \\
\hline Senecio olivaceobracteatus & & 1,8 & & & & 5,1 & 0,5 & & & 1,8 \\
\hline Tagetes multiflora & 3,8 & 1,8 & 0,8 & 0,6 & 0,6 & & & 0,9 & 1,0 & \\
\hline Baccharis petiolata & 2,3 & & & & & 5,1 & & & & \\
\hline Balbisia microphylla & & & & & & & & 5,4 & 1,0 & 0,9 \\
\hline Gnaphalium glandulosum & 1,5 & 1,8 & 3,1 & & & & & & & \\
\hline Jarava leptostachya & & & & & & & & & & 5,9 \\
\hline Calceolaria inamoena & 0,8 & 1,8 & & & & 2,1 & 0,5 & & & \\
\hline Ephedra breana & & 3,6 & & & & 0,5 & & 0,5 & & \\
\hline Fuertesimalva chilensis & 3,0 & & 0,8 & 0,6 & 0,6 & & & & & \\
\hline Nassella pubiflora & 5,3 & & & & & & & & & \\
\hline Tunilla soehrensii & & 5,4 & & & & & & & & \\
\hline Coreopsis suaveolens & & & & & & & & & & 4,1 \\
\hline Lupinus oreophilus & 0,8 & & 3,1 & & & & & & & \\
\hline Spergularia fasciculata & & & & 1,2 & & & & 0,9 & 1,5 & 0,5 \\
\hline Dunalia spinosa & & & & & & 3,1 & & & & \\
\hline Mutisia acuminata & & & & & & 3,1 & & & & \\
\hline Senecio behnii & 3,0 & & & & & & & 0,5 & & \\
\hline Bartsia bartsioides & & & & & & & 1,0 & 0,5 & & 0,5 \\
\hline Senna birostris & & & & & & & & 0,5 & 1,5 & \\
\hline Cardionema ramosissimum & & & & 1,2 & & & 0,5 & & & \\
\hline Conyza deserticola & & & 1,6 & & & & & & & 0,5 \\
\hline Poa sp. & 1,5 & & & & & & & & & \\
\hline Tetraglochin cristatum & & & & & & 0,5 & & & & 0,9 \\
\hline Bowlesia tropaeolifolia & 0,8 & & & & & & & & & \\
\hline Bromus catharticus & 0,8 & & & & & & & & & \\
\hline Pellaea ternifolia & & & & 1,2 & & & & & & \\
\hline Quinchamalium parviflorum & & & & & & 0,5 & & & & \\
\hline Cumulopuntia boliviana & & & & & & & & & 0,5 & \\
\hline Oreocereus variicolor & 0,8 & & & & & & & & & \\
\hline Total $(\%)$ & 100 & 100 & 100 & 100 & 100 & 100 & 100 & 100 & 100 & 100 \\
\hline Cobertura vegetal absoluta (\%) & 67 & 57 & 68 & 57,7 & 62,7 & 76,7 & 69,7 & 75,3 & 66,7 & 79,0 \\
\hline Índice de Shannon & 2,3 & 1,7 & 2,4 & 1,4 & 1,9 & 2,6 & 2,0 & 2,3 & 2,4 & 2,6 \\
\hline Riqueza de especies & 19 & 15 & 15 & 13 & 13 & 20 & 15 & 18 & 19 & 23 \\
\hline
\end{tabular}


TABLA IV. Cobertura vegetal absoluta y relativa (\%), riqueza de especies e índice de biodiversidad de Shannon en las parcelas de muestreo durante la estación seca.

TABLE IV. Absolute and relative vegetal cover (\%), species richness and Shannon index of biodiversity in samplyng sites during dry season.

\begin{tabular}{|c|c|c|c|c|c|c|c|c|c|c|}
\hline \multirow[b]{2}{*}{ Especie / Parcela } & \multicolumn{3}{|c|}{ Año 2002} & \multicolumn{7}{|c|}{ Año 2003} \\
\hline & 1 & 2 & 3 & 4 & 5 & 6 & 7 & 8 & 9 & 10 \\
\hline Fabiana ramulosa & 35 & 18,1 & 31,3 & 26,4 & 0,7 & 28,1 & 30,3 & 14,7 & & 5,5 \\
\hline Baccharis boliviensis & 18,8 & 13,9 & 19,4 & 35,5 & 11,0 & 12,8 & 16,1 & 13,6 & 20,6 & 15,8 \\
\hline Diplostephium meyenii & 18,8 & 25,0 & 3,0 & 16,4 & & 17,9 & 17,4 & 27,1 & 19,9 & 2,4 \\
\hline Poaceae & & 7,0 & 3,0 & 1,8 & 6,2 & 5,1 & 13,6 & 8,5 & 17,8 & 9,1 \\
\hline Lophopappus tarapacanus & 5,0 & & & 7,3 & & 4,3 & & 22,0 & 23,3 & 1,8 \\
\hline Parastrephia lepidophylla & & & & & 61,7 & & & & & \\
\hline Baccharis santelicis & & 4,2 & 16,4 & 0,9 & 5,5 & 1,7 & & 4,0 & 3,4 & 7,3 \\
\hline Adesmia spinosissima & & 1,4 & 6,0 & & 8,2 & 1,3 & 12,9 & 6,2 & 2,1 & 3,6 \\
\hline Polylepis rugulosa & & & 6,0 & & & & & & & 35,3 \\
\hline Verbena gynobasis & 1,3 & & & 6,4 & 2,7 & 15,3 & 3,9 & & 4,1 & \\
\hline Senecio olivaceobracteatus & & 12,5 & & 0,9 & & 3,8 & & & 2,1 & 1,8 \\
\hline Plantago sericea & & 1,4 & 11,9 & & & & 3,9 & & & \\
\hline Jarava leptostachya & 7,5 & 4,2 & & 2,7 & & & & & & \\
\hline Plazia daphnioides & & & & & & & & & & 12,7 \\
\hline Balbisia microphylla & 8,8 & & & & & 0,4 & & 2,3 & & \\
\hline Ephedra breana & 1,3 & 8,3 & & & & & & 1,7 & & \\
\hline Cumulopuntia sphaerica & & 1,4 & & 1,8 & & & 1,9 & & 4,1 & 1,2 \\
\hline Calceolaria inamoena & & 1,4 & & & & 3,0 & & & 2,1 & \\
\hline Lupinus oreophilus & & 1,4 & 3,0 & & & & & & & \\
\hline Stipa $\mathrm{sp}$ & & & & & 4,1 & & & & & \\
\hline Mutisia acuminata & & & & & & 3,8 & & & & \\
\hline Coreopsis suaveolens & & & & & & & & & & 3,6 \\
\hline Diplostephium cinereum & & & & & & 2,6 & & & & \\
\hline Krameria lapaccea & 2,5 & & & & & & & & & \\
\hline Adesmia verrucosa & 1,3 & & & & & & & & & \\
\hline Senna birostris & & & & & & & & & 0,7 & \\
\hline Total $(\%)$ & 100 & 100 & 100 & 100 & 100 & 100 & 100 & 100 & 100 & 100 \\
\hline Cobertura vegetal absoluta (\%) & 40 & 37 & 32,5 & 37,7 & 47,3 & 81 & 52,7 & 60 & 49,7 & 55 \\
\hline Indice de Shannon & 1,8 & 2,1 & 1,9 & 1,7 & 1,3 & 2,1 & 1,8 & 1,9 & 1,9 & 2,0 \\
\hline Riqueza de especies & 10 & 13 & 9 & 10 & 8 & 13 & 8 & 9 & 11 & 12 \\
\hline
\end{tabular}

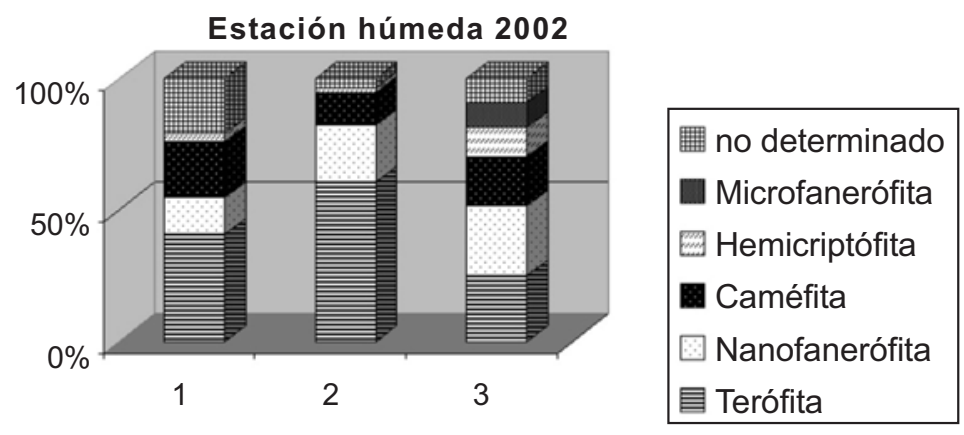

Parcela

FIGURA 4: Cobertura vegetal según forma de vida, durante la estación húmeda del 2002.

FiguRE 4: Vegetal cover by life form in wet season 2002. 


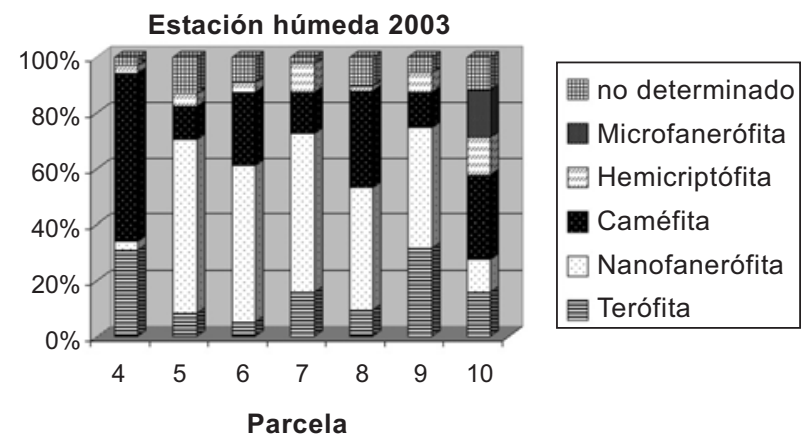

Figura 5: Cobertura vegetal según forma de vida, durante la estación húmeda del 2003.

Figure 5. Vegetal cover by life form in wet season 2003.

TABLA V. Cobertura vegetal (\%) según forma de vida durante la estación húmeda y seca.

TABLE V. Vegetal cover (\%) by life form during wet and dry season.

\begin{tabular}{llll|llllllll}
\hline \multicolumn{10}{c}{ Parcelas } \\
\hline & \multicolumn{1}{c}{ Año 2002} & \multicolumn{1}{c}{ Año 2003} \\
\hline Forma de vida & 1 & 2 & 3 & 4 & 5 & 6 & 7 & 8 & 9 & 10 \\
\hline Terófita & 40,6 & 60,7 & 25,0 & 31,1 & 8,2 & 5,1 & 16,1 & 9,5 & 31,8 & 15,9 \\
Nanofanerófita & 14,3 & 21,4 & 26,6 & 3,0 & 62,1 & 55,9 & 56,6 & 43,9 & 42,9 & 11,8 \\
Caméfita & 21,1 & 12,5 & 18,8 & 59,9 & 12,1 & 26,2 & 14,6 & 34,8 & 12,6 & 30,0 \\
Hemicriptófita & 3,0 & 1,8 & 10,9 & 3,0 & 4,4 & 3,6 & 10,3 & 1,8 & 7,1 & 13,6 \\
Microfanerófita & & & 9,4 & & & & & & & 16,8 \\
no determinado & 21,1 & 3,6 & 9,4 & 3,0 & 13,2 & 9,2 & 2,4 & 10,4 & 5,6 & 11,8 \\
\hline Nanofanerófita* & 68,8 & 59,7 & 43,3 & 50,9 & 70,6 & 65,1 & 60,7 & 72,3 & 50,0 & 27,9 \\
Caméfita* & 23,8 & 27,8 & 35,8 & 44,5 & 19,2 & 29,8 & 21,9 & 19,2 & 32,2 & 27,9 \\
Hemicriptófita* & 7,5 & 5,6 & 11,9 & 2,7 & 4,1 & & 3,9 & & & 35,2 \\
Microfanerófita* & & & 6,0 & & & & & & & \\
no determinado* & 7,0 & 3,0 & 1,8 & 6,2 & 5,1 & 13,6 & 8,5 & 17,8 & 9,1 \\
\hline
\end{tabular}

( ): estación húmeda

$(*)$ : estación seca

\section{RIQUEZA Y DIVERSIDAD}

La riqueza de especies presente en los transectos realizados en las parcelas durante la estación seca fue siempre menor a los realizados en igual parcela durante la estación húmeda. Durante la estación seca el promedio de riqueza fue igual a 10,3 $\pm 1,9$ especies . Durante la estación húmeda la riqueza fue igual a $17 \pm 3,3$ especies de plantas vasculares. Así, según la prueba por rangos de Spearman, la cobertura vegetal y la riqueza de especies en un área determinada presentan una relación positiva $\left(\mathrm{r}_{\mathrm{s}}=\right.$ $0,745, \mathrm{n}=20 ; \mathrm{P}=0,01)$, y según el test de significancia de la prueba, la relación es altamente significativa $\left(\mathrm{t}_{18}=6,33 ; \mathrm{P}=0,01\right)$.
Respecto a la relación de abundancia de especies, el índice de Shannon arrojó mayores valores, por ende mayor biodiversidad, en las parcelas medidas durante la estación húmeda, excepto en dos de las 10 parcelas.

\section{VEGETACIÓN}

Para la definición de formaciones vegetacionales los valores de cobertura de plantas agrupadas según tipo biológico fueron convertidos a los códigos propuestos por la metodología de la Carta de Ocupación de Tierras. Los resultados se muestran en la Tabla VI. 
TABLA VI. Descripción de formaciones vegetacionales del área de estudio.

TABLA VI. Description of vegetation formations in the study area.

\begin{tabular}{|c|c|c|c|c|c|}
\hline Parcela & $\begin{array}{l}\text { Formación } \\
\text { Vegetal }\end{array}$ & $\begin{array}{c}\text { Código } \\
\text { estación seca }(*)\end{array}$ & $\begin{array}{l}\text { Especies dominantes } \\
\text { estación seca }(* *)\end{array}$ & $\begin{array}{c}\text { Código } \\
\text { estación húmeda }(*)\end{array}$ & $\begin{array}{l}\text { Especies dominantes } \\
\text { estación húmeda }(* *)\end{array}$ \\
\hline 1 & Tolar & $\mathrm{LB}_{4} / \mathrm{H}_{1}$ & $\begin{array}{c}\text { LB: Fr-Dm-Bb } \\
\text { H: Sl }\end{array}$ & $\mathrm{LB}_{3} / \mathrm{H}_{3} / \mathrm{H}_{3} / \mathrm{S}_{1}$ & $\begin{array}{c}\text { LB: Bb-Fr } \\
\text { H: “p”-S sp. } \\
\text { H: Ph-Mg } \\
\text { S: Cs }\end{array}$ \\
\hline 2 & Tolar & $\mathrm{LB}_{4} / \mathrm{H}_{1} / \mathrm{H}_{1} / \mathrm{S}_{1}$ & $\begin{array}{c}\text { LB: Dm-Fr-Bb } \\
\text { H: “p”-Sl } \\
\text { H: Ps } \\
\text { S: Cs }\end{array}$ & $\mathrm{LB}_{3} / \mathrm{H}_{1} / \mathrm{H}_{4} / \mathrm{S}_{1}$ & $\begin{array}{l}\text { LB: Dm } \\
\text { H: "p" } \\
\text { H: Ph } \\
\text { S: Cb }\end{array}$ \\
\hline 3 & Queñoal & $\mathrm{LA}_{1} / \mathrm{LB}_{3} / \mathrm{H}_{1} / \mathrm{H}_{1}$ & $\begin{array}{c}\text { LA: Pr } \\
\text { LB: Fr-Bb-Bs } \\
\text { H: “p” } \\
\text { H: Ps }\end{array}$ & $\mathrm{LA}_{2} / \mathrm{LB}_{4} / \mathrm{H}_{1} / \mathrm{H}_{4}$ & $\begin{array}{c}\text { LA: Pr } \\
\text { LB: Fr-Bb-Bs } \\
\text { H: "p" } \\
\text { H: Cc-Ps }\end{array}$ \\
\hline 4 & Tolar & $\mathrm{LB}_{4} / \mathrm{H}_{1} / \mathrm{S}_{1}$ & $\begin{array}{l}\text { LB: Bb-Fr-Dm } \\
\text { H: “p”-Sl } \\
\text { S: Cs }\end{array}$ & $\mathrm{LB}_{4} / \mathrm{H}_{1} / \mathrm{H}_{3} / \mathrm{S}_{1}$ & $\begin{array}{l}\text { LB: Bb } \\
\text { H: “p” } \\
\text { H: Ph } \\
\text { S: Cs }\end{array}$ \\
\hline 5 & Tolar & $\mathrm{LB}_{4} / \mathrm{H}_{2}$ & $\begin{array}{l}\text { LB: Pl-Bb-As } \\
\text { H: “p”-S sp. }\end{array}$ & $\mathrm{LB}_{4} / \mathrm{H}_{2} / \mathrm{H}_{1} / \mathrm{S}_{1}$ & $\begin{array}{l}\text { LB: Pl-Fr-Bb } \\
\text { H: “p”-Sl } \\
\text { H: Mg } \\
\text { S: Cs }\end{array}$ \\
\hline 6 & Tolar & $\mathrm{LB}_{5} / \mathrm{H}_{1}$ & $\begin{array}{c}\text { LB: Fr-Dm-Vg } \\
\text { H: "p" }\end{array}$ & $\mathrm{LB}_{5} / \mathrm{H}_{2} / \mathrm{H}_{3} / \mathrm{S}_{1}$ & $\begin{array}{l}\text { LB: Vg-Fr-Dm } \\
\text { H: “p”-S sp. } \\
\text { H: Ph-Se sp. } \\
\text { S: Cs }\end{array}$ \\
\hline 7 & Tolar & $\mathrm{LB}_{4} / \mathrm{H}_{1} / \mathrm{H}_{1} / \mathrm{S}_{1}$ & $\begin{array}{c}\text { LB: Fr-Dm-Bb } \\
\text { H: "p" } \\
\text { H: Ps } \\
\text { S: Cs }\end{array}$ & $\mathrm{LB}_{4} / \mathrm{H}_{1} / \mathrm{H}_{3} / \mathrm{S}_{1}$ & $\begin{array}{c}\text { LB: Fr-Dm-Bb } \\
\text { H: “p” } \\
\text { H: Ph-Ps } \\
\text { S: Cs }\end{array}$ \\
\hline 8 & Tolar & $\mathrm{LB}_{5} / \mathrm{H}_{3} / \mathrm{H}_{1}$ & $\begin{array}{c}\text { LB: Dm-Lt-Fr-Bb } \\
\text { H: "p" } \\
\text { H: Eb }\end{array}$ & $\mathrm{LB}_{5} / \mathrm{H}_{2} / \mathrm{H}_{2} / \mathrm{S}_{1}$ & $\begin{array}{c}\text { LB: Bb-Fr-Dm } \\
\text { H: “p” } \\
\text { H: Ph } \\
\text { S: Cs }\end{array}$ \\
\hline 9 & Tolar & $\mathrm{LB}_{5} / \mathrm{H}_{3} / \mathrm{S}_{1}$ & $\begin{array}{c}\text { LB: Lt-Bb-Dm } \\
\text { H: “p” } \\
\text { S: Cs }\end{array}$ & $\mathrm{LB}_{4} / \mathrm{H}_{2} / \mathrm{H}_{3} / \mathrm{S}_{1}$ & $\begin{array}{l}\text { LB: Dm-Lt-Bb } \\
\text { H: “p”-S sp. } \\
\text { H: Ph-Mp-Mg } \\
\text { S: Cb-Cs }\end{array}$ \\
\hline 10 & Queñoal & $\mathrm{LA}_{5} / \mathrm{LB}_{4} / \mathrm{H}_{2} / \mathrm{S}_{1}$ & $\begin{array}{l}\text { LA: Pr } \\
\text { LB: Bb-Ph } \\
\text { H: “p” } \\
\text { S: Cs }\end{array}$ & $\mathrm{LA}_{3} / \mathrm{LB}_{4} / \mathrm{H}_{2} / \mathrm{H}_{3} / \mathrm{S}_{1}$ & $\begin{array}{c}\text { LA: } \mathrm{Pr} \\
\text { LB: Bs-Bb } \\
\text { H: “p”-S1 } \\
\text { H: Ph-Ps } \\
\text { S: Cs }\end{array}$ \\
\hline
\end{tabular}

(*) Estratificación tipo biológico: $\mathrm{LA}=2-4 \mathrm{~m} ; \mathrm{LB}=0.5-1 \mathrm{~m} ; \mathrm{H}=0.5-1 \mathrm{~m} ; \mathrm{H}=0-25 \mathrm{~cm} ; \mathrm{S}=1-2 \mathrm{~m} ; \mathrm{S}=0-25 \mathrm{~cm}$ Cobertura tipo biológico: $1=1-5 \% ; 2=>5-10 \% ; 3=>10-25 \% ; 4=>25-50 \% ; 5=>50-75 \%$

(**) As: Adesmia spinosissima, Bb: Baccharis boliviensis, Bs: Baccharis santelicis, Cb: Cumulopuntia boliviana, Cc: Cremolobus chilensis, Cs: Cumulopuntia sphaerica, Eb: Ephedra breana, Dm: Diplostephium meyenii, Fr: Fabiana ramulosa, Lt: Lophopappus tarapacanus, Mg: Microsteris gracilis, Mp: Muhlenbergia peruviana, Ov: Oreocereus variicolor, "p":Poaceae, Ph: Plantago hispidula, Pd: Plazia daphnoides, Pl: Parastrephia lepidophylla, Pr: Polylepis rugulosa, Ps: Plantago sericea, Sl: Stipa leptostachya, S sp.: Stipa sp., Se sp.: Senecio sp., Vg: Verbena gynobasis 
En general, según los tipos de crecimiento y su participación, se distinguen dos formaciones vegetacionales:

A) TOLAR: comunidad vegetal presente en laderas de cerros, compuesta principalmente por arbustos resinosos tanto de hábito perenne o caducifolio de la familia Asteraceae, tales como Baccharis boliviensis, Diplostephium meyenii, Lophopappus tarapacanus, Parastrephia lepidophylla, y Solanaceae (Fabiana ramulosa). Entre ellos se insertan algunas cactáceas (e.g. Cumulopuntia spp., Oreocereus spp.) y gramíneas cespitosas, principalmente de los géneros Festuca y Stipa. En los espacios de suelo desnudo entre los arbustos y a continuación de las lluvias, aparece una estrata herbácea, ya sea de especies terófitas (e.g. Plantago hispidula, Tagetes multiflora, Muhlenbergia peruviana) o aquellas perennes que sólo asoman parte aérea ante las más favorables condiciones hídricas (e.g. Cardionema ramosissimum), con lo cual aumenta la cobertura vegetal del suelo, incluso superando el 70 $\%$. En esta formación vegetacional se distinguieron dos asociaciones: Baccharis boliviensis-Fabiana ramulosa-Diplostephium meyenii sensu Villagrán (parcelas uno, dos, cuatro, seis, ocho, nueve) y Parastrephia lepidophylla-Baccharis boliviensisFabiana ramulosa (parcela cinco). La primera de ellas corresponde a la formación vegetacional de mayor distribución en el área de estudio. No obstante, dentro del tolar es posible encontrar otras diferencias en la distribución de especies; respecto a la orientación de la ladera, los "viejitos", cactáceas columnares del género Oreocereus, ocurren principalmente en laderas solanas; en el caso de Cumulopuntia sphaerica ("perrito"), que estando presente en laderas de distinta exposición, aumenta notoriamente su cobertura en laderas escarpadas de mayor exposición solar. Otras especies del tolar son más abundantes en terrenos arenosos, frecuentemente en bordes de camino como Baccharis scandens, Mutisia acuminata, Dunalia spinosa y Senna birostris var. arequipensis.

b) QUEÑOAL: la principal diferencia con el tolar es justamente la presencia de la queñoa (Polylepis rugulosa) agregando la estrata arbórea a la formación vegetacional. Comúnmente se trata de individuos de entre 2 a $4 \mathrm{~m}$ de altura con abundancia relativa variable generalmente no superior al $40 \%$. Bajo y entre la queñoa se desarrolla una comunidad vegetal arbustiva como la descrita anteriormente. Las parcelas tres y 10 fueron clasificadas dentro de esta formación vegetacional. Esta comunidad podría analogarse a la asociación Polylepis rugulosaFabiana ramulosa señalada por Rundel (2003).

\section{DISCUSION}

El área de estudio constituye un valioso ejemplo de la vegetación altoandina en el norte de Chile; la precordillera en esta latitud está considerada como la región vegetacional más diversa, tanto en riqueza como cobertura, de los Andes del norte de Chile con régimen de lluvias estivales, vale decir entre aproximadamente 18 y $24^{\circ} \mathrm{S}$ (Villagrán et al. 1981, Arroyo et al. 1988, Gutiérrez et al. 1988). En el caso particular de las especies leñosas, según Rundel et al. (2003) el área de estudio posee la comunidad de plantas leñosas más diversa del norte de Chile.

En cuanto a la flora del área, es destacable el alto porcentaje que es de origen nativo, lo cual podría explicarse en parte por las rigurosas características físicas en la zona producto de la altura; otros autores han señalado una menor riqueza de plantas exóticas en zonas altas, tanto en Chile (Pauchard \& Alaback 2004), como en otros continentes (Wilson et al. 1992). Por otra parte, la baja densidad poblacional respecto a otras zonas del país podría traducirse en falta de propágulos de especies exóticas, los cuales llegan usualmente ligados a actividades humanas (Sakai et al. 2001, Figueroa et al. 2004).

Es relevante mencionar el caso de la única especie monocotiledónea no gramínea, Olsynium scirpoideum subsp. scirpoideum (Iridaceae). Esta geófita no estaba registrada en la I Región, sino otra iridácea de tépalos amarillos, Olsynium trinerve (Baker) R.A. Rodr. et Martic. (Gajardo 1997, Rodríguez \& Marticorena 2000). La muestra recolectada en el área de estudio, de tépalos rosados, fue enviada a la Universidad de Concepción y reconocida por Rodríguez como $O$. scirpoideum, planta que fue vista en laderas de cerros cercanas al camino que une Putre y Zapahuira y en los alrededores de Chapiquiña en sitios restringidos.

Dentro de las especies clasificadas como naturalizadas merece atención el caso de Cheilanthes cf. bonariensis según Marticorena y Rodríguez (1995), esta especie presenta una amplia distribución en el continente americano desde Estados Unidos, a lo largo de la Cordillera de los Andes hasta el norte de Argentina; sin embargo en 
Chile sólo se ha visto en la costa de la provincia de Antofagasta. Elizabeth Barrera (Museo Nacional de Historia Natural de Chile) reconoció la muestra como Cheilanthes bonariensis siguiendo las claves presentes en dicho trabajo, pero recomendó confrontar con otras muestras identificadas. Parece luego cuestionable afirmar que la especie es naturalizada.

En general, la identidad y riqueza de especies vegetales vasculares encontradas en el área de estudio ( $\mathrm{n}=98$ especies), así como la proporción de sus formas de vida, son similares a las descritas por Villagrán et al. (1981) en un estudio realizado en la provincia de Parinacota. Respecto a las formas de vida, la alta preponderancia de caméfitas, nanofanerófitas y hemicriptófitas no es de extrañar considerando ciertas características de ellas. Caméfitas y hemicriptófitas son formas de vida especializadas para vivir en ambientes extremos debido a que presentan yemas de renuevo a nivel de suelo o cercano a él (Gajardo 1997). Respecto a las nanofanerófitas, y leñosos bajos en general, su presencia en ambientes áridos se ve favorecida por la capacidad de producir sistemas radicales grandes y profundos que les permiten obtener humedad durante una estación más prolongada (Rundel et al. 2003). También se presentan características adaptativas particulares a ciertas especies como tamaño de hoja reducido (e.g. Fabiana ramulosa, Parastrephia lepidophylla, Baccharis boliviensis, Balbisia microphylla), hojas resinosas (Lophoppapus tarapacanus, Parastrephia lepidophylla, Coreopsis suaveolens) y metabolismo ácido crasuláceo (CAM) en el caso de especies de la familia Cactaceae, una de las familias con mayor número de especies.

La forma de vida menos representada corresponde a las microfanerófitas con una especie, Polylepis rugulosa. La queñoa (nombre vernacular) es el único árbol nativo del área de estudio y, además de algunos individuos aislados de Eucalyptus sp., constituye el único recurso maderero. En efecto, la queñoa lleva una larga trayectoria de sobreexplotación (Negrete 1997, Marquet et al. 1998) y Polylepis besseri Hieron., revalidada para Chile como Polylepis rugulosa (Kessler 1995), está considerada como una especie vulnerable (Benoit 1989).

Tanto la flora como la vegetación del área de estudio arrojaron diferencias a través de un gradiente estacional. Los datos concuerdan con la literatura en que luego de la caída de lluvias aumenta la riqueza de especies y la cobertura vegetal del suelo; este aumento de cobertura sobrepasó en forma común el $60 \%$, lo cual discrepa con lo observado por Villagrán et al. (1982), aunque únicamente en un corto período.

Al agrupar la abundancia de especies según formas de vida se ratifican los patrones estacionales. Como es de esperar, a excepción de un par de meses a continuación de la estación lluviosa, la forma de vida terófitas está ausente al igual que hemicriptófitas herbáceas, que permanecen latentes bajo el suelo esperando el siguiente período de mayor disponibilidad hídrica. En efecto, la relación positiva entre cobertura y número de especies concuerda con que el aumento de cobertura vegetal es explicable, al menos en parte, por la aparición de otras especies. Durante la estación seca dominan nanofanerófitas y caméfitas, que en su mayoría corresponden a arbustos o tolas. La representatividad de la totalidad de la flora en los transectos es baja, y al reparar en los datos de cobertura vegetal relativa se aprecia cómo pocas especies, y las mismas, ocupan gran parte de la cobertura vegetal relativa; Este es el caso de Baccharis boliviensis, Fabiana ramulosa, Diplostephium meyenii y Lophoppapus tarapacanus, lo cual desde un punto de vista de tipo biológico es una dominancia de leñosos bajos, formando el paisaje de la estepa arbustiva prealtiplánica (sensu Gajardo). La especie aquí referida como Fabiana ramulosa (basiónimo Fabiana densa var. ramulosa Wedd.) ha sido anteriormente indentificada en asociaciones vegetales como Fabiana densa (Villagrán et al. 1981, 1982; Arroyo et al. 1988).

Aunque la cobertura de la única especie microfanerófita es variable, su presencia fue criterio de categorización en otra asociación por el ostensible cambio en la fisionomía del paisaje vegetal al incluir un nuevo tipo biológico y otra estrata vegetal en altura. Los "queñoales" se encontraban generalmente en faldeos de cerros con poca pendiente o en el fondo de quebradas.

La diversidad de los sitios de muestreo también mostró variaciones estacionales. Para interpretar los índices de diversidad es importante mencionar que incorporan tanto la riqueza de especies como su ocurrencia o abundancia relativa (Ludwig \& Reynolds, 1988). La mayor diversidad ocurrida en la mayoría de las parcelas durante la estación húmeda puede explicarse en el aumento del número de especies. En el caso 
excepcional de las parcelas dos y cuatro, el efecto de aumento de especies fue contrarrestado por lo poco equitativo de la repartición de coberturas entre especies en la estación húmeda respecto a la misma parcela durante la estación seca. La parcela dos, si bien durante la estación húmeda posee mayor número de especies, su cobertura está monopolizada en $53,6 \%$ por una sola especie herbácea: Plantago hispidula, siendo la distribución de cobertura entre las especies menos equitativa que durante la estación seca. En la parcela cuatro ocurrió algo similar; habiendo más especies durante la estación húmeda, ésta presentó mayor participación de una sola especie y la gran mayoría del resto de las especies (ocho de 12 restantes) presentan una baja participación (inferior al 2\%). Así mismo, la parcela cinco muestra un valor de biodiversidad notoriamente bajo durante la estación seca. En esta parcela una sola especie, Parastrephia lepidophylla, representa más del $60 \%$ de la cobertura vegetal durante la estación mencionada.

Las formaciones vegetacionales encontradas en el área de estudio han sido similarmente reportadas en otros trabajos (Villagrán et al. 1981, 1982; Rundel et al. 2003). La comunidad dominada por la especie Parastrephia lepidophylla es esperada de encontrar en las partes más altas del tolar (sobre $3.700 \mathrm{~m}$ de altitud), en una comunidad en transición con el siguiente piso vegetacional, el pajonal, en donde la diversidad es más restringida (Villagrán et al. 1981; CORFO 1982). En la parcela 5, dominada por esta especie, efectivamente se encontró una menor biodiversidad.

En un contexto interanual, si bien las mediciones de cobertura vegetal realizadas durante la estación húmeda fueron mayores a las realizadas durante la estación seca en ambos años de muestreo, el cambio proporcional en cobertura entre estaciones fue mayor en el año en que el monto de lluvias estivales fue normal y muy superior al registrado entre diciembre de 2002 a marzo del año siguiente. Esto podría indicar que el aumento de cobertura vegetal es dependiente de la cantidad de agua caída, sin embargo, la diferencia no fue significativa estadísticamente. Es, luego, destacable la gran adaptabilidad a la sequía de estas comunidades vegetales por mostrar su estacionalidad en cobertura y composición de especies vegetales, aún cuando las precipitaciones fueron alrededor de un tercio de la pluviometría promedio registrada en el área (período 1993-2002, DGA-MOP).

Entre la diversidad vegetal, las terófitas fueron la comunidad de plantas más afectada por la disminución en las precipitaciones. Cabe destacar que las terófitas, o hierbas anuales, no sólo son sensibles a la disponibilidad hídrica, sino que, según Belmonte (comunicación personal), la población de terófitas en precordillera ha disminuido debido al aumento de la masa de ganado introducido en los últimos años.

Para concluir, en estudios futuros sería interesante estudiar como las terrazas de cultivo, creadas hace miles de años y que en los últimos años han sido abandonadas, se han ido recolonizando de especies nativas.

\section{AGRADECIMIENTOS}

Los autores desean agradecer a Wild Life Trust y al Proyecto Macs (Manejo sostenible de camélidos sudamericanos silvestres) ICA4-2000-10299, sin cuyo financiamiento este estudio no hubiese sido posible. También deseamos agradecer encarecidamente la colaboración de Mélica Muñoz y Elizabeth Barrera, ambas de la Sección Botánica del Museo Nacional de Historia Natural, en el reconocimiento de las especies vegetales y el acceso a literatura especializada.

\section{BIBLIOGRAFIA}

Aceituno, P. 1997. Aspectos generales del clima en el Altiplano sudamericano. En: Actas del II Simposio Internacional de Estudios Altiplánicos: El Altiplano, ciencia y conciencia en los Andes, pp. 63-70. Universidad de Chile. Santiago, Chile.

Arroyo, M.T.K., F.A. Squeo, J.J. Armesto \& C. VILLAGRÁN. 1988. Effects of aridity on plant diversity in the northern Chilean Andes: results of a natural experiment. Annals of the Missouri Botanical Garden 75: 55-78.

Arroyo, M.T.K., F. Squeo, H. Veit, L. Cavieres, P. León \& E. Belmonte. 1997. Flora and Vegetation of Northern Chilean Andes. En: Actas del II Simposio Internacional de Estudios Altiplánicos. El Altiplano, ciencia y conciencia en los Andes, pp. 167-178. Universidad de Chile. Santiago, Chile.

Barboza, G. \& A.T. Hunziker. 1993. Estudios en Solanaceae XXXIV. Revisión taxonómica de Fabiana. Kurtziana 22: 109-153. 
Variación estacional en vegetación andina. MuÑoz, A. \& C. BonACiC

Benort I.L.1989. Libro Rojo de la Flora Terrestre de Chile. CONAF, Santiago. 157 pp.

Cavieres, L.A., A. Peñaloza \& M. Kalin Arroyo. 2000. Pisos altitudinales de vegetación en los Andes de Chile central $\left(33^{\circ} \mathrm{S}\right)$. Revista Chilena de Historia Natural 73: 331-344.

Conaf. 1981. Delimitación y caracterización de los ecosistemas de la I Región. Corporación Nacional Forestal: Gerencia de Desarrollo, Santiago. 88 pp.

Corfo. 1982. Análisis de los Ecosistemas de la I región. Sociedad Agrícola CORFO Ltda., Universidad de Chile, Santiago. 194 pp.

ConAf. 1986. Plan de Manejo del Parque Nacional Lauca. Corporación Nacional Forestal, Santiago.

Charrier, R. 1997. Ciencias de la tierra y recursos mineros y energéticos en el Altiplano chileno. En: Actas del II Simposio Internacional de Estudios Altiplánicos: El Altiplano, ciencia y conciencia en los Andes, pp. 5-14. Universidad de Chile. Santiago, Chile.

Di CAStRi, F. \& E.R. HAJek. 1976. Bioclimatología de Chile. Editorial de la Universidad Católica de Chile, Santiago. 129 pp.

Etienne, M. \& C. Prado. 1982. Descripción de la vegetación mediante la cartografía de ocupación de tierras. Ciencias Agrícolas Nº10, Universidad de Chile, Santiago. 120 pp.

Figueroa, J.A., S.A. Castro, P.A. Marquet \& F.M. Jaksic. 2004. Invasión de plantas exóticas en la región mediterránea de Chile: causas, historia e impactos. Revista Chilena de Historia Natural 77: 465-483.

GAJARDO. M. 1997. Caracterización florística de diferentes ambientes de la Región de Tarapacá (I Región, Chile). Tesis Ingeniero Agrónomo, Universidad de Chile, Chile.

Gajardo, R. 1994. La Vegetación Natural de Chile. Clasificación y Distribución Geográfica. Editorial Universitaria, Santiago. 165 pp.

Glade. A. 1993. Libro Rojo de los Vertebrados Terrestres de Chile. Conaf. 2a edición. Ministerio de Agricultura, Santiago. 68 pp.

Gutiérrez, J.R., F. López-Cortés \& P.A. Marquet. 1988. Vegetation in an altitudinal gradient along the Río Loa in the Atacama Desert of northern Chile. Journal of arid environments 40: 383-399.

Heady H.F., R.P. Gibbens \& R.W. Powell. 1959. A Comparison of the Charting, Line Intercept, and Line Point Methods of Sampling Shrub Types of Vegetation. Journal of Range Management 12: 180188.

Hoffmann A.E. \& H.E. Walter. 2004. Cactáceas en la Flora Silvestre de Chile. Ediciones Fundación Claudio Gay. $2^{\text {a }}$ edición. Santiago. 307 pp.

Hunt, D. 1999. Cites Cactaceae Check list. Second edition. Royal Botanic Gardens Kew \& International Organization for Succulent Plant Study (IOS), Milborne Port, $315 \mathrm{pp}$.

Kent, M. \& P. CoOKer. 1992. Vegetation description and analysis, a practical approach. John Wiley \& Sons, New York. 363 pp.
Kessler, M. 1995. Revalidación de Polylepis rugulosa Bitter (Rosaceae). Gayana Botánica 52: 49-51.

Ludwig, J.A. \& J.F. Reynolds. 1988. Statistical Ecology, a primer on methods and computing. John Wiley \& Sons, Inc., USA. 337 pp.

Marquet, P.A., F. Bozinovic, G.A. Bradwhaw, C. Cornelius, H. González, J.L. Gutiérrez, E.R. HajeK, J.A. Lagos, F. López-Cortés, L. Núnez, E.F. Rosello, C. Santoro, H. Samaniego, V.G. Standen, J.C. Torres-Mura \& F.M. Jaksic. 1998. Los ecosistemas del Desierto de Atacama y área andina adyacente en el norte de Chile. Revista Chilena de Historia Natural 71: 593-617.

Marticorena, C. 1990. Contribución a la estadística de la flora vascular de Chile. Gayana Botánica 47: 85113.

Marticorena, C. \& M. Quezada.1985. Catálogo de la flora vascular de Chile. Gayana Botánica 42:1-157.

Marticorena, C. \& R. Rodríguez. 1995. Flora de Chile. Volumen I: Pterydophyta-Gimnospermae. Universidad de Concepción, Concepción. 351 pp.

Negrete, R. 1997. La Vegetación en el Altiplano. En: Actas del II Simposio Internacional de Estudios Altiplánicos. El Altiplano, ciencia y conciencia en los Andes, pp. 161-166. Universidad de Chile. Santiago, Chile.

Novoa, R. \& Villaseca. 1989. Mapa agroclimático de Chile. Instituto de Investigaciones Agropecuarias, Santiago. 126 pp.

Pauchard, A. \& P. Alaback. 2004. Influence of Elevation, Land Use, and Landscape Context on Patterns of Alien Plant Invasions along Roadsides in Protected Areas of South-Central Chile. Conservation Biology 18: 238-248.

Rodríguez, R. \& C. Marticorena. 2000. Comentarios taxonómicos en Iridáceas chilenas. Gayana Botánica 57 (2):169-179.

Rundel, P.W., A.C. Gibson, G.S. Midgley, S.J.E. Wand, B. Palma, C. Kleier \& J. Lambrinos. 2003. Ecological and ecophysiological patterns in a prealtiplano shrubland of the Andean Cordillera in northern Chile. Plant Ecology 169: 179-193.

SaKai, A.K., F.W. Allendorf, J.S. Holt, D.M. Lodge, J. Molofsky, K.A. With, S. Baughman, R.J. Cabin, J.E. Cohen, N.C. Ellstrand, de McCcauley, P. O’Neil, M.I. Parker, J.N. Thompson \& S.G. WELLER. 2001. The population biology of invasive species. Annual Review of Ecology and Systematics 32: 305-332.

Sielfeld, W., C. Carrasco, G. González, J. Torres, A. CAREvic \& I. Ilanino. 1988. Estudio de la taruca (Hippocamelus antisensis) en Chile. Informe final Proyecto CONAF/PNUD7FAO-CHI/83/017, Arica. 97 pp.

Sielfeld, W., C. Carrasco, G. GonzÁlez \& J. Torres. 1999. La taruca (Hippocamelus antisensis D'Orbigny 1834, Cervidae, Artiodactyla) en la provincia de Parinacota, Región de Tarapacá, Chile: Población, hábitat y alimentación. Anales del Museo de Historia Natural de Valparaíso 24: 95-108. 
Gayana Bot. 63(1), 2006

Soreng, R.J. (Ed.). 2003. Catalogue of New World Grasses (Poaceae): IV. Subfamily Pooideae. Smithsonian Institution. Contributions from the United States National Herbarium 48: 1-730.

Soreng, R.J. \& S.J. Pennington (Eds.). 2003. Catalogue of New World Grasses (Poaceae): III. Subfamilies Panicoideae, Aristidoideae, Arundinoideae, and Danthonioideae. Smithsonian Institution. Contributions from the United States National Herbarium 46: 1-62.

SutherLAND, W.J. 1996. Ecological Census Techniques, a handbook. Cambridge University Press, United Kingdom. 336 pp.

Troncoso, R. 1983. Caracterización ambiental del ecosistema bofedal de Parinacota y su relación con la vegetación. Tesis de Ingeniero Agrónomo, Universidad de Chile, Chile.
Villagrán, C., J.J. Armesto \& M.T.K. Arroyo. 1981. Vegetation in a high Andean transect between Turi and Cerro León in northern Chile. Vegetatio 48: 3-16.

Villagrán, C., M.T.K. Arroyo \& J. Armesto. 1982. La vegetación de un transecto altitudinal en los Andes del norte de Chile (18-19 $\mathrm{S})$. En: El ambiente natural y las poblaciones humanas de los Andes del norte grande de Chile (Arica, Lat.18 $28^{\prime} \mathrm{S}$ ) (Eds. A.Veloso \& E. Bustos), pp.13-70. UNESCO. Santiago.

Wilson, J.B., G.L. Rapson, M.T. Sykes, A.J. Watkins \& P.A. Williams. 1992. Distribution and climatic correlations of some exotic species along roadsides in South Islands, New Zealand. Journal of Biogeography 19: 183-193. 